\title{
Activated anilide in heterocyclic synthesis: Synthesis of new hydrazo, dihydropyridazine, tetrahydropyridine, dihydropyridine and pyranopyridine derivatives
}

\author{
IBRAHIM SAAD ABDEL HAFIZ ${ }^{\mathrm{a}, *}$, MAHMOUD MOHAMED MAHFOUZ RAMIZ ${ }^{\mathrm{b}}$ and \\ MOHAMED AHMED ELIAN ${ }^{\mathrm{a}}$ \\ ${ }^{a}$ Department of Chemistry, Faculty of Education, Suez Canal University, Arish 45511, Egypt \\ ${ }^{b}$ Department of Physics and Mathematical Engineering, Faculty of Electronic Engineering, \\ Minoufyia University, Menouf 32952, Egypt \\ e-mail: dr_ibrahim.saad@yahoo.com
}

MS received 16 September 2011; revised 26 December 2011; accepted 11 January 2012

\begin{abstract}
A series of new hydrazo, dihydropyridazine, tetrahydropyridine, dihydropyridine and pyranopyridine derivatives with known biological activity have been prepared through the reactions of 3-oxo-3-phenyl-N(pyridine-3-yl) propanamide $\mathbf{3}$ and enaminonitrile $\mathbf{1 7}$ with some electrophilic reagents, nucleophilic reagents, and aryl diazonium salts. The newly synthesized compounds were characterized by IR, ${ }^{1} \mathrm{H}$ NMR and mass spectral studies.
\end{abstract}

Keywords. Hydrazo; dihydropyridazine; tetrahydropyridine; dihydropyridine; pyranopyridine.

\section{Introduction}

The pyridine nucleus is one of the most important heterocycles found in many natural products, pharmaceuticals and functional materials. ${ }^{1}$ Several substituted pyridines and their derivatives were reported to exhibit significant antimicrobial, ${ }^{2}$ antiinflammatory ${ }^{3}$ and anticancer activities. ${ }^{4}$ Other evidence for the potent activity of pyridine in biological systems is its presence in the important vitamins niacin and pyridoxine (vitamin B6) and also in highly toxic alkaloids such as nicotine. ${ }^{5-7}$ The wide-ranging biological activity associated with many substituted pyridine derivatives, both naturally occurring and synthetic, ensures that the synthesis of this important ring system remains a topic of current interest.

During the past decades increasing interest in the synthesis and biological activities of pyridazine derivatives has been observed. ${ }^{8-10}$ Pyridazine compounds have been reported to possess varied biological activities such as anticonvulsant, ${ }^{9}$ antibacterial, ${ }^{11}$ antiinflammatory ${ }^{12}$ anticancer $^{13}$ and antiplatelet ${ }^{14}$ activities. These facts have prompted us to synthesize some novel pyridazine derivatives. Recently, pyridazinone nucleus has been extensively studied in the search for new and selective medicinal agents as drugs acting on the cardiovascular system. ${ }^{15,16}$

*For correspondence
There has been considerable interest in the development of novel compounds with anticonvulsant, antidepressant, analgesic, antiinflammatory, antiplatelet, antimalarial, antimicrobial, antimycobacterial, antitumoral, vasodilator, antiviral and antischistosomiasis activities. Hydrazones possessing an azometine $-\mathrm{NHN}=\mathrm{CH}-$ proton constitute an important class of compounds for new drug development. Therefore, many researchers have synthesized these compounds as target structures and evaluated their biological activities. ${ }^{17-25}$ These observations have been guiding factors for the development of new hydrazones that possess expected biological activities, because we have been involved in a program aimed at developing new rout for the synthesis of heterocyclic compounds of biological interest. ${ }^{26,27}$ In previous studies, we reported the utility of polyfunctionally substituted heterocycles in heterocyclic synthesis. ${ }^{28,29}$ In continuation of this work and as apart of our biological chemistry programme we report here the utility of 3-oxo-3-phenyl-N-(pyridine-3-yl) propanamide 3 and enaminonitrile $\mathbf{1 7}$ in the synthesis of a wide variety of unique heterocyclic systems with expected biological activities.

\section{Experimental}

Melting points were determined using a Büchi apparatus and are uncorrected. The purity of compound was 
confirmed by TLC using Merck silica gel $60 \mathrm{~F}_{254}$ plates using toluene, ethyl acetate and methanol as a mobile phase and spots were visualized under UV radiation. IR spectra $(\mathrm{KBr})$ were recorded on a Bruker-Vector 22 instrument (Bruker) and frequencies are expressed in $\mathrm{cm}^{-1}$. NMR spectra were recorded with a Varian Gemini spectrometer $(300 \mathrm{MHz}$ and $200 \mathrm{MHz}$ ) with TMS as the internal reference. Chemical shifts were reported on a ppm scale $(\delta)$ relative to TMS as a standard. EI-mass spectra were recorded with a Shimadzu Qp-2010 plus. Elemental analyses were performed at the Microanalytical Centre at the Faculty of Science, Cairo University, Egypt.

\subsection{Preparation of 3-oxo-3-phenyl- $\mathrm{N}$ - (pyridin-3-yl)propanamide (3)}

A mixture of 3-aminopyridine ( $10 \mathrm{mmol}$ ) and ethyl benzoylacetate $(10 \mathrm{mmol})$ was refluxed in xylene $(30 \mathrm{~mL})$ for $5 \mathrm{~h}$. The reaction mixture was allowed to cool and the separated solid product was collected by filtration and crystallized from ethanol to give compound $\mathbf{3}$ as white crystals, $85 \%$ Yield; mp $100-102^{\circ} \mathrm{C}$; IR (KBr) $3424(\mathrm{NH}), 3050$ (CH-arom), 2923 (CH-aliph), 1685 and $1718(\mathrm{C}=\mathrm{O}) \mathrm{cm}^{-1} ;{ }^{1} \mathrm{H}$ NMR $\left(\mathrm{CDCl}_{3}\right) \delta 4.16(\mathrm{~s}$, $\left.2 \mathrm{H}, \mathrm{CH}_{2}\right), 7.24-8.70(\mathrm{~m}, 9 \mathrm{H}$, aromatic $\mathrm{H}), 9.67$ (s, $1 \mathrm{H}, \mathrm{NH}) ;{ }^{13} \mathrm{C} \mathrm{NMR}\left(\mathrm{CDCl}_{3}\right) \delta 45.10,123.25,124.60$, $127.8,127.8,128.2,128.2,132.2,135.25,135.90$, 137.3, 153.20,172.5, 191.70. MS $(\mathrm{m} / \mathrm{z}) 240\left(\mathrm{M}^{+}\right)$, Anal. Calcd. for $\mathrm{C}_{14} \mathrm{H}_{12} \mathrm{~N}_{2} \mathrm{O}_{2}$ : C, 69.99; H, 5.03; N, 11.66; Found: C, 69.94; H, 5.01; N, 11.61.

\subsection{General procedure for the synthesis of hydrazo derivatives $\mathbf{5 a - d}$}

A cold suspension of aryl diazonium salts $4 \mathbf{a}-\mathbf{d}$ $(10 \mathrm{mmol})$ (prepared from $10 \mathrm{mmol}$ of aryl aromatic amine with the appropriate quantities of sodium nitrite and hydrochloric acid) was gradually added to a cold solution $\left(0-5^{\circ} \mathrm{C}\right)$ of $3(10 \mathrm{mmol})$ in ethanol $(50 \mathrm{~mL})$ containing sodium acetate $(2 \mathrm{~g})$ with continuous stirring for $1 \mathrm{~h}$. The resulting reaction product was filtered off, washed with water and crystallized from the proper solvent to give compounds $\mathbf{5 a - d .}$

2.2a 3-Oxo-3-phenyl-2-(2-phenylhydrazono)- $N$-(pyridin3-yl)propanamide (5a): Formed as yellow crystals (ethanol); 85\% Yield; mp 150-152 ${ }^{\circ} \mathrm{C}$; IR (KBr) 3363 and $3128(\mathrm{NH}), 3050$ (CH-arom), 1716 and 1655 $(\mathrm{C}=\mathrm{O}) \mathrm{cm}^{-1} ;{ }^{1} \mathrm{H}$ NMR $\left(\mathrm{CDCl}_{3}\right) \delta 7.14-8.90(\mathrm{~m}, 14 \mathrm{H}$, aromatic $\mathrm{H}), 11.77(\mathrm{~s}, 1 \mathrm{H}, \mathrm{NH}), 14.72(\mathrm{~s}, 1 \mathrm{H}, \mathrm{NH})$; ${ }^{13} \mathrm{C} \mathrm{NMR}\left(\mathrm{CDCl}_{3}\right) \delta 116.00,116.00,125.00,125.55$,
126.70, 126.70, 129.40, 129.40,131.40, 131.40, 132.95, $134.20,135.40,135.90,137.60,146.5,149.20,154.50$, 160.00, 192.90. MS $(\mathrm{m} / \mathrm{z}) 344\left(\mathrm{M}^{+}\right)$, Anal. Calcd. for $\mathrm{C}_{20} \mathrm{H}_{16} \mathrm{~N}_{4} \mathrm{O}_{2}$ : C, 69.76; H, 4.68; N, 16.27; Found: C, $69.71 ; \mathrm{H}, 4.63 ; \mathrm{N}, 16.23$.

2.2b 2-(2-(4-Chlorophenyl)hydrazono)-3-oxo-3-phenyl$\mathrm{N}$-(pyridin-3-yl)propanamide $(\mathbf{5} \boldsymbol{b})$ : Formed as pale yellow crystals (ethanol); $88 \%$ Yield, mp $178-180^{\circ} \mathrm{C}$; IR (KBr) 3311 and 3142 (NH), 3070 (CH-arom), 1608 and $1658(\mathrm{C}=\mathrm{O}) \mathrm{cm}^{-1} ;{ }^{1} \mathrm{H}$ NMR $\left(\mathrm{CDCl}_{3}\right) \delta 7.13-$ $8.44(\mathrm{~m}, 13 \mathrm{H}$, aromatic $\mathrm{H}), 8.88(\mathrm{~s}, 1 \mathrm{H}, \mathrm{NH}), 11.70$ (s, 1H, NH); MS $(m / z) 378\left(\mathrm{M}^{+}\right)$, Anal. Calcd. for $\mathrm{C}_{20} \mathrm{H}_{15} \mathrm{ClN}_{4} \mathrm{O}_{2}$ : C, 63.41; H, 3.99; Cl, 9.36; N, 14. 79; Found: C, 63.38; H, 3.94; Cl, 9.31; N, 14.74.

2.2c 3-Oxo-3-phenyl-N-(pyridin-3-yl)-2-(2-p-tolylhydrazono)propanamide (5c): Formed as dark yellow crystals (ethanol); $82 \%$ Yield; mp $175-177^{\circ} \mathrm{C}$; IR (KBr) 3337 and $3116(\mathrm{NH}), 3050(\mathrm{CH}$-arom), $2921(\mathrm{CH}-$ aliph), 1617 and $1649(\mathrm{C}=\mathrm{O}) \mathrm{cm}^{-1} ;{ }^{1} \mathrm{H}$ NMR $\left(\mathrm{CDCl}_{3}\right)$ $\delta 2.34(\mathrm{~s}, 3 \mathrm{H}, \mathrm{Me}), 7.10-8.43(\mathrm{~m}, 13 \mathrm{H}$, aromatic $\mathrm{H})$, 8.91 (s, 1H, NH), 11.82 (s, 1H, NH); MS $(m / z) 358$ $\left(\mathrm{M}^{+}\right)$, Anal. Calcd. for $\mathrm{C}_{21} \mathrm{H}_{18} \mathrm{~N}_{4} \mathrm{O}_{2}$ : C, 70.38; H, 5.06; N, 15. 63; Found: C, 70.33; H, 5.01; N, 15.58 .

2.2d 2-(2-(4-Methoxyphenyl)hydrazono)-3-oxo-3phenyl-N-(pyridin-3-yl)propanamide (5d): Formed as dark yellow crystals (ethanol/DMF); 90\% Yield; mp 210-212 ${ }^{\circ} \mathrm{C}$; IR (KBr) 3285 and $3150(\mathrm{NH}), 3090$ (CH-arom), 2934 (CH-aliph), 1634 and $1668(\mathrm{C}=\mathrm{O})$ $\mathrm{cm}^{-1} ;{ }^{1} \mathrm{H}$ NMR $\left(\mathrm{CDCl}_{3}\right) \delta 3.82(\mathrm{~s}, 3 \mathrm{H}, \mathrm{MeO}), 6.89$ $9.10(\mathrm{~m}, 13 \mathrm{H}$, aromatic $\mathrm{H}), 12.02(\mathrm{~s}, 1 \mathrm{H}, \mathrm{NH}), 14.81$ (s, 1H, NH); MS $(m / z) 374\left(\mathrm{M}^{+}\right)$, Anal. Calcd. for $\mathrm{C}_{21} \mathrm{H}_{18} \mathrm{~N}_{4} \mathrm{O}_{3}$ : C, 67.37; H, 4.85; N, 14. 96; Found: C, $67.32 ; \mathrm{H}, 4.80 ; \mathrm{N}, 14.91$.

\subsection{General procedure for the synthesis} of dihydropyridazine derivatives $7 \boldsymbol{a}-\boldsymbol{d}$

A mixture of compounds 5a-d (10 mmol), ammonium acetate $(10 \mathrm{mmol})$ and malononitrile $(10 \mathrm{mmol})$ was fused in domestic microwave oven for $3 \mathrm{~min}$. The solid precipitate so formed was treated with ethanol and filtered out and crystallized from the proper solvent.

2.3a 5-Cyano-6-imino-1,4-diphenyl-N-(pyridin-3-yl)-1, 6-dihydropyridazine-3-carboxamide (7a): Formed as red-brown crystals (ethanol); 52\% Yield; mp 160$162^{\circ} \mathrm{C}$; IR ( $\left.\mathrm{KBr}\right) 3441$ and $3298(\mathrm{NH}), 3100(\mathrm{CH}-$ arom), $2196(\mathrm{CN}), 1625(\mathrm{C}=\mathrm{O}) \mathrm{cm}^{-1},{ }^{1} \mathrm{H} \mathrm{NMR}$ 
$\left(\mathrm{CDCl}_{3}\right) \delta 7.26-8.01(\mathrm{~m}, 15 \mathrm{H}$, arom-H and $\mathrm{NH}$ group), $10.00(\mathrm{~s}, 1 \mathrm{H}, \mathrm{NH}) ;{ }^{13} \mathrm{C}$ NMR $\left(\mathrm{CDCl}_{3}\right) \delta 107.75$, 123.00, 124.70, 124.70, 124.95 125.25, 127.30, 128.90, $128.90,129.90,129.90,131.00,131.00,132.30$, 133.90, 135.2, 137.50, 141.00, 144.45, 149.50, 152.00, 158.50, 164.80. MS $(\mathrm{m} / \mathrm{z}) 392\left(\mathrm{M}^{+}\right)$, Anal. Calcd. for $\mathrm{C}_{23} \mathrm{H}_{16} \mathrm{~N}_{6} \mathrm{O}$ : C, 70.04; H, 4.11; N, 21.42; Found: C, 69.97; H, 4.09; N, 21.27.

2.3b 1-(4-Chlorophenyl)-5-cyano-6-imino-4-phenyl- $N$ (pyridin-3-yl)-1,6-dihydropyridazine-3-carboxamide $(7 \boldsymbol{b})$ : Formed as pale yellow crystals (ethanol); 57\% Yield; mp 188-190 ${ }^{\circ}$; IR (KBr) 3315 and 3207 (NH), 3049 (CH-arom), $2206(\mathrm{CN}), 1648(\mathrm{C}=\mathrm{O}) \mathrm{cm}^{-1} ;{ }^{1} \mathrm{H}$ NMR $\left(\mathrm{CDCl}_{3}\right) \delta 6.90-7.88(\mathrm{~m}, 14 \mathrm{H}$, arom-H and $\mathrm{NH}$ group), 10.12 (s, 1H, NH); MS $(m / z) 426\left(\mathrm{M}^{+}\right)$; Anal. Calcd. for $\mathrm{C}_{23} \mathrm{H}_{15} \mathrm{ClN}_{6} \mathrm{O}: \mathrm{C}, 64.72 ; \mathrm{H}, 3.54 ; \mathrm{Cl}, 8.31$; N, 19.60; Found: C, 64.38; H, 3.50; Cl; 8.24; N, 19.55 .

2.3c 5-Cyano-6-imino-4-phenyl-N-(pyridin-3-yl)-1-ptolyl-1,6-dihydropyridazine-3-carboxamide (7c): Formed as red-brown crystals (ethanol); 54\% Yield; $\mathrm{mp} 170-172^{\circ} \mathrm{C}$; IR (KBr) 3272 and 3128 (NH), 2921(CH-aliph), $2197(\mathrm{CN}), 1626(\mathrm{C}=\mathrm{O}) \mathrm{cm}^{-1} ;{ }^{1} \mathrm{H}$ NMR $\left(\mathrm{CDCl}_{3}\right) \delta 2.30$ (s, 3H, Me); 7.10-7.92 (m, 14H, arom-H and $\mathrm{NH}$ group), 9.87 (s, 1H, NH); Anal. Calcd. for $\mathrm{C}_{24} \mathrm{H}_{18} \mathrm{~N}_{6} \mathrm{O}: \mathrm{C}, 70.92 ; \mathrm{H}, 4.46$; N, 20.68; Found: $\mathrm{C}$, $70.89 ; \mathrm{H}, 4.41 ; \mathrm{N}, 20.61$.

2.3d 5-Cyano-6-imino-1-(4-methoxyphenyl)-4-phenyl$N$-(pyridin-3-yl)-1,6-dihydro-pyridazine-3-carboxamide (7d): Formed as red-brown crystals (dioxane); 58\% Yield; mp 178-180 ${ }^{\circ}$; IR (KBr) 3300 and 3285 (NH), 3064 (CH-arom), 2921(CH-aliph), $2201(\mathrm{CN}), 1647$ $(\mathrm{C}=\mathrm{O}) \mathrm{cm}^{-1} ;{ }^{1} \mathrm{H}$ NMR $\left(\mathrm{CDCl}_{3}\right) \delta 3.80(\mathrm{~s}, 3 \mathrm{H}, \mathrm{MeO})$; 7.30-8.12 (m, 14H, arom-H and $\mathrm{NH}$ group), 10.22 (s, $1 \mathrm{H}, \mathrm{NH})$; MS $(\mathrm{m} / z) 424\left(\mathbf{M}^{+}+2\right)$; Anal. Calcd. for $\mathrm{C}_{24} \mathrm{H}_{18} \mathrm{~N}_{6} \mathrm{O}_{2}$ : C, 68.24; H, 4.29; N, 19.89; Found: C, $68.11 ; \mathrm{H}, 4.15 ; \mathrm{N}, 19.70$.

\subsection{General procedure for the synthesis of tetrahydropyridine derivatives $10 a-d$}

A mixture of propanamide derivatives $3(10 \mathrm{mmol})$ and arylidenemalononitriles $\mathbf{1 0 a}-\mathbf{d}(10 \mathrm{mmol})$ in ethanol $(100 \mathrm{~mL})$ containing catalytic amount of piperidine was heated under reflux for $5 \mathrm{~h}$. The reaction mixture was allowed to cool and poured into crushed ice then acidified with $\mathrm{HCl}$. The separated solid was filtered, washed with water and crystallized from the proper solvent to give the tetrahydropyridine derivative 10a-d. 2.4a 2-Amino-5-benzoyl-6-oxo-4-phenyl-1-(pyridin3-yl)-1,4,5,6-tetrahydropyridine-3-carbonitrile (10a): Formed as white crystals (dioxane); $85 \%$ Yield; mp 243-245 ${ }^{\circ}$; IR (KBr) 3250, $3194\left(\mathrm{NH}_{2}\right), 3064(\mathrm{CH}-$ arom), 2934 (CH-aliph), $2187(\mathrm{CN}), 1699$ and 1654 $(\mathrm{C}=\mathrm{O}) \mathrm{cm}^{-1} ;{ }^{1} \mathrm{H} \mathrm{NMR}\left(\mathrm{CDCl}_{3}\right) \delta 4.25(\mathrm{~d}, 1 \mathrm{H}, J=6.6$ $\mathrm{Hz}, \mathrm{H}-4), 4.92$ (d, 1H, J = 6.6 Hz, H-5), 7.26-8.14 (m, $14 \mathrm{H}$, aromatic $\mathrm{H}), 8.78\left(\mathrm{~s}, 2 \mathrm{H}, \mathrm{NH}_{2}\right) ;{ }^{13} \mathrm{C} \mathrm{NMR}$ $\left(\mathrm{CDCl}_{3}\right) \delta$ 37.95, 68.60, 83.80, 115.40, 122.50, $126.80,127.40,127.40,128.35,129.2$, 129.2, 129.60, $129.60,131.70,131.70,135.30,138.50,140.00$, $140.90,141.35,148.70,157.40,169.10,194.70$. Anal. Calcd. for $\mathrm{C}_{24} \mathrm{H}_{18} \mathrm{~N}_{4} \mathrm{O}_{2}$ : C, 73.08; H, 4.60; N, 14.20; Found: C, 73.02; H, 4.52; N, 14.16.

2.4b 2-Amino-5-benzoyl-4-(4-chlorophenyl)-6-oxo-1(pyridin-3-yl)-1,4,5,6-tetrahydropyridine-3-carbonitrile $(\mathbf{1 0 b})$ : Formed as white crystals (dioxane); $60 \%$ Yield; mp 238-240 ${ }^{\circ}$; IR (KBr) 3250, $3165\left(\mathrm{NH}_{2}\right)$, 3050 (CH-arom), 2923 (CH-aliph), $2178(\mathrm{CN}), 1702$ and $1685(\mathrm{C}=\mathrm{O}) \mathrm{cm}^{-1} ;{ }^{1} \mathrm{H}$ NMR $\left(\mathrm{DMSO}-\mathrm{d}_{6}\right) \delta 4.32(\mathrm{~d}$, $1 \mathrm{H}, J=8.1 \mathrm{~Hz}, \mathrm{H}-4), 5.35(\mathrm{~d}, 1 \mathrm{H}, J=8.1 \mathrm{~Hz}, \mathrm{H}-5)$, 7.42-8.61 (m, $13 \mathrm{H}$, aromatic $\mathrm{H}), 8.62\left(\mathrm{~s}, 2 \mathrm{H}, \mathrm{NH}_{2}\right)$; MS $(m / z) 428\left(\mathrm{M}^{+}\right)$, Anal. Calcd. for $\mathrm{C}_{24} \mathrm{H}_{17} \mathrm{ClN}_{4} \mathrm{O}_{2}$ : C, 67.21; H, 4.00; Cl, 8.27; N, 13.06; Found: C, 67.17; $\mathrm{H}, 3.95 ; \mathrm{Cl}, 8.22 ; \mathrm{N}, 13.01$.

2.4c 2-Amino-5-benzoyl-4-(2-chlorophenyl)-6-oxo-1(pyridin-3-yl)-1,4,5,6-tetrahydropyridine-3-carbonitrile (10c): Formed as white crystals (dioxane); 78\% Yield; mp 214-216 ${ }^{\circ}$; IR (KBr) 3343, $3250\left(\mathrm{NH}_{2}\right)$, 3073 (CH-arom), 2954 (CH-aliph), $2194(\mathrm{CN}), 1690$ and $1655(\mathrm{C}=\mathrm{O}) \mathrm{cm}^{-1} ;{ }^{1} \mathrm{H}$ NMR $\left(\mathrm{DMSO}_{-} \mathrm{d}_{6}\right) \delta 4.62(\mathrm{~d}$, $1 \mathrm{H}, J=6.6 \mathrm{~Hz}, \mathrm{H}-4), 4.87$ (d, $1 \mathrm{H}, J=6.6 \mathrm{~Hz}, \mathrm{H}-5)$, $7.27-8.68(\mathrm{~m}, 13 \mathrm{H}$, aromatic $\mathrm{H}), 8.75\left(\mathrm{~s}, 2 \mathrm{H}, \mathrm{NH}_{2}\right)$; Anal. Calcd. for $\mathrm{C}_{24} \mathrm{H}_{17} \mathrm{ClN}_{4} \mathrm{O}_{2}$ : C, 67.21; H, 4.00; Cl, 8.27; N, 13.06; Found: C, 67.18; H, 3.95; Cl, 8.23; N, 13.02 .

2.4d 2-Amino-5-benzoyl-4-(2,4-dichlorophenyl)-6-oxo1-(pyridin-3-yl)-1,4,5,6-tetrahydropyridine-3-carbonitrile (10d): Formed as brown crystals (ethanol); $70 \%$ Yield; mp 140-142 ${ }^{\circ} \mathrm{C}$; IR (KBr) 3349, $3337\left(\mathrm{NH}_{2}\right)$, 3086 (CH-arom), 2960 (CH-aliph), $2210(\mathrm{CN}), 1680$ and $1635(\mathrm{C}=\mathrm{O}) \mathrm{cm}^{-1} ;{ }^{1} \mathrm{H}$ NMR (DMSO-d $\left.\mathrm{d}_{6}\right) \delta 4.55$ $(\mathrm{d}, 1 \mathrm{H}, J=6.6 \mathrm{~Hz}, \mathrm{H}-4), 4.79(\mathrm{~d}, 1 \mathrm{H}, J=6.6$ $\mathrm{Hz}, \mathrm{H}-5), 7.18-8.54(\mathrm{~m}, 12 \mathrm{H}$, aromatic $\mathrm{H}), 8.98$ (s, $\left.2 \mathrm{H}, \mathrm{NH}_{2}\right)$; $\mathrm{MS}(m / z) 465\left(\mathrm{M}^{+}+2\right)$, Anal. Calcd. for $\mathrm{C}_{24} \mathrm{H}_{16} \mathrm{Cl}_{2} \mathrm{~N}_{4} \mathrm{O}_{2}$ : C, 62.22; H, 3.48; $\mathrm{Cl}, 15.30 ; \mathrm{N}, 12.09$; Found: C, 62.18; H, 3.43; Cl, 15.25; N, 12.04 . 


\subsection{General procedure for the synthesis} of tetrahydropyridine derivative $16 a, b$

A mixture of propanamide $3(10 \mathrm{mmol})$ and 2(ethoxymethylene) malononitrile 13a $(10 \mathrm{mmol})$ or ethyl 2-cyano-3-ethoxyacrylate $\mathbf{1 3 b}(10 \mathrm{mmol})$ in ethanol $(100 \mathrm{~mL})$ containing catalytic amount of piperidine was refluxed for $7 \mathrm{~h}$. The reaction mixture was allowed to cool and poured into crushed ice then acidified with $\mathrm{HCl}$, the solid product so formed was collected by filtration and crystallized from the proper solvent.

2.5a 2-Amino-5-benzoyl-4-ethoxy-6-oxo-1-(pyridin-3yl)-1,4,5,6-tetrahydropyridine-3-carbonitrile (16a): Formed as brown crystals (dioxane); 65\% Yield; mp 232-234 ${ }^{\circ}$; IR (KBr) 3348, $3182\left(\mathrm{NH}_{2}\right), 2948(\mathrm{CH}-$ aliph), $2213(\mathrm{CN}), 1620$ and $1641(\mathrm{C}=\mathrm{O}) \mathrm{cm}^{-1} ;{ }^{1} \mathrm{H}$ NMR (DMSO-d $\left.{ }_{6}\right) \delta 1.55(\mathrm{t}, 3 \mathrm{H}, J=8.5 \mathrm{~Hz}, \mathrm{Me}), 2.88$ $\left(\mathrm{q}, 2 \mathrm{H}, \mathrm{CH}_{2}\right), 4.20(\mathrm{~d}, 1 \mathrm{H}, J=5.2 \mathrm{~Hz}, \mathrm{H}-4), 5.19$ (d, $1 \mathrm{H}, J=5.2 \mathrm{~Hz}, \mathrm{H}-5), 7.35-8.51(\mathrm{~m}, 9 \mathrm{H}$, aromatic $\mathrm{H})$, $8.61\left(\mathrm{~s}, 2 \mathrm{H}, \mathrm{NH}_{2}\right) ;{ }^{13} \mathrm{C} \mathrm{NMR}\left(\mathrm{CDCl}_{3}\right) \delta$ 18.70, 58.20, $66.30,66.40,83.60,108.30,122.60,128.30,129.50$, $129.50,131.60,131.60,134.90,137.00,138.00$, 138.50, 145.70, 156.00, 167.90, 195.90, MS $(\mathrm{m} / \mathrm{z}) 362$ $\left(\mathrm{M}^{+}\right)$, Anal. Calcd. for $\mathrm{C}_{20} \mathrm{H}_{18} \mathrm{~N}_{4} \mathrm{O}_{3}$ : C, 66.29; $\mathrm{H}, 5.01$; N, 15.46; Found: C, 66.25; H, 4.98; N, 15.41.

2.5b Ethyl-2-amino-5-benzoyl-4-ethoxy-6-oxo-1-(pyridin3-yl)-1,4,5,6-tetrahydropyridine-3-carboxylate (16b): Formed as pale brown crystals (ethanol/dioxane); $70 \%$ Yield; mp 176-178 ${ }^{\circ}$; IR (KBr) 3425, $3148\left(\mathrm{NH}_{2}\right)$, 3029 (CH-arom), 2973 (CH-aliph), 1611 and 1710 and $1740(\mathrm{C}=\mathrm{O}) \mathrm{cm}^{-1} ;{ }^{1} \mathrm{H}$ NMR $\left(\right.$ DMSO-d $\left._{6}\right) \delta 1.20(\mathrm{t}, 3 \mathrm{H}$, $J=5.8 \mathrm{~Hz}, \mathrm{Me}), 1.60(\mathrm{t}, 3 \mathrm{H}, J=6.1 \mathrm{~Hz}, \mathrm{Me}), 3.00$ (q, 2H, $\left.\mathrm{CH}_{2}\right), 3.84$ (q, 2H, $\left.\mathrm{CH}_{2}\right), 4.19$ (d, 1H, $J=5.2$ $\mathrm{Hz}, \mathrm{H}-4), 5.33$ (d, 1H, J = 5.2 Hz, H-5), 7.04-8.77 $(\mathrm{m}, 9 \mathrm{H}$, aromatic $\mathrm{H}), 8.78\left(\mathrm{~s}, 2 \mathrm{H}, \mathrm{NH}_{2}\right)$; $\mathrm{MS}(\mathrm{m} / z) 409$ $\left(\mathrm{M}^{+}\right)$, Anal. Calcd. for $\mathrm{C}_{22} \mathrm{H}_{23} \mathrm{~N}_{3} \mathrm{O}_{5}$ : C, 64.54; H, 5.66; N, 10.26; Found: C, 64.49; H, 5.61; N, 10.21.

2.6 Preparation of 2-ethyl-4-methyl-6-oxo-1, 6dihydropyridine-3-carbonitrile (21)

A mixture of $\beta$-enaminonitrile $17(10 \mathrm{mmol})$ and ethyl acetoacetate $(10 \mathrm{mmol})$ was refluxed in xylene $(30 \mathrm{~mL})$ for $7 \mathrm{~h}$. The reaction mixture was allowed to cool and the separated solid product was collected by filtration and crystallized from ethanol to give compound $\mathbf{2 1}$ as pale yellow crystals (ethanol/dioxane); $70 \%$ Yield; mp 136-138 ${ }^{\circ}$; IR (KBr) 3330 (NH), 2973 (CH-aliph), $2220(\mathrm{CN}), 1661(\mathrm{C}=\mathrm{O}) ;{ }^{1} \mathrm{H}$ NMR $\left(\mathrm{CDCl}_{3}\right) \delta 1.19(\mathrm{~s}$, $3 \mathrm{H}, \mathrm{Me}$ ), 1.29 (t, 3H, $J=4.5 \mathrm{~Hz}, \mathrm{Me}$ ), 2.66 (q, 2H,
$\mathrm{CH}_{2}$ ), 4.99 (br, 1H, NH), 6.10 (s, 1H, H-pyridine); MS $(m / z) 164\left(\mathbf{M}^{+}+2\right)$, Anal. Calcd. for $\mathrm{C}_{9} \mathrm{H}_{10} \mathrm{~N}_{2} \mathrm{O}: \mathrm{C}$, 66.65; H, 6.21; N, 17.27; Found: C, 66.60; H, 6.18; N, 17.22 .

\subsection{General procedure for the synthesis} of dihydropyridine derivatives $\mathbf{2 2 a} \boldsymbol{b}$

A cold suspension of aryl diazonium salts $\mathbf{4 b , c}$ $(10 \mathrm{mmol})$ was gradually added to a cold solution $(0$ $\left.5^{\circ} \mathrm{C}\right)$ of $21(10 \mathrm{mmol})$ in ethanol $(50 \mathrm{~mL})$ containing anhydrous sodium acetate $(2 \mathrm{~g})$ with continuous stirring for $1 \mathrm{~h}$. The resulting reaction product was filtered off, washed with water and crystallized from the proper solvent to give compounds 22a,b

2.7a 5-((4-Chlorophenyl)diazenyl)-2-ethyl-4-methyl6-oxo-1,6-dihydropyridine-3 carbonitrile (22a): Formed as red brown crystals (ethanol); 78\% Yield; mp 100-102 ${ }^{\circ} \mathrm{C}$; IR (KBr) 3435 (NH), 2921 (CH-aliph), $2200(\mathrm{CN}), 1680(\mathrm{C}=\mathrm{O}) \mathrm{cm}^{-1} ;{ }^{1} \mathrm{H}$ NMR $\left(\mathrm{CDCl}_{3}\right)$ $\delta 1.18(\mathrm{t}, 3 \mathrm{H}, J=4.7 \mathrm{~Hz}, \mathrm{Me}), 2.15(\mathrm{~s}, 3 \mathrm{H}, \mathrm{Me})$, 2.81 (q, 2H, $\left.\mathrm{CH}_{2}\right), 7.22-7.88(\mathrm{~m}, 4 \mathrm{H}$, arom-H), 8.01 $(\mathrm{s}, 1 \mathrm{H}, \mathrm{NH}) ;{ }^{13} \mathrm{C} \mathrm{NMR}\left(\mathrm{CDCl}_{3}\right) 12.30,18.00,23.5$, 83.20, 110.10, 112.80, 123.70, 123.70, 124.00, 130.90, 130.90, 144.60, 153.40, 155.20, 185.60. MS $(m / z) 301$ $\left(\mathrm{M}^{+}+1\right)$, Anal. Calcd. for $\mathrm{C}_{15} \mathrm{H}_{13} \mathrm{ClN}_{4} \mathrm{O}: \mathrm{C}, 59.91 ; \mathrm{H}$, 4.36; Cl, 11.79; N, 18.63; Found: C, 59.88; H, 4.32; Cl, $11.74 ; \mathrm{N}, 18.58$.

$2.7 \mathrm{~b}$ 2-Ethyl-4-methyl-6-oxo-5-(p-tolyldiazenyl)-1,6dihydropyridine-3-carbonitrile (22b): Formed as brown crystals (ethanol); $87 \%$ Yield; mp $101-103^{\circ} \mathrm{C}$; IR (KBr) $3342(\mathrm{NH}), 2921$ (CH-aliph), $2196(\mathrm{CN})$, $1627(\mathrm{C}=\mathrm{O}) \mathrm{cm}^{-1} ;{ }^{1} \mathrm{H}$ NMR $\left(\mathrm{CDCl}_{3}\right) \delta 2.20(\mathrm{~s}, 3 \mathrm{H}$, $\mathrm{Me}), 2.42$ (s, 3H, Me), 1.22 (t, 3H, $J=4.5 \mathrm{~Hz}, \mathrm{Me}$ ), $2.78\left(\mathrm{q}, 2 \mathrm{H}, \mathrm{CH}_{2}\right), 7.15-7.90(\mathrm{~m}, 4 \mathrm{H}$, arom-H), 8.00 (s, 1H, NH); MS $(m / z) 282\left(\mathrm{M}^{+}+2\right)$, Anal. Calcd. for $\mathrm{C}_{16} \mathrm{H}_{16} \mathrm{~N}_{4} \mathrm{O}: \mathrm{C}, 68.55 ; \mathrm{H}, 5.75 ; \mathrm{N}, 19.99$; Found: C, $68.50 ; \mathrm{H}, 5.72 ; \mathrm{N}, 19.94$.

\subsection{General procedure for preparation of pyranopyridine derivatives $\mathbf{2 7} \boldsymbol{a}, \boldsymbol{b}$}

A mixture of 2-ethyl-4-methyl-6-oxo-1,6-dihydropyridine-3-carbonitrile $\mathbf{2 1}(10 \mathrm{mmol})$ and arylidenemalononitriles $\mathbf{8 b}, \mathbf{c}(10 \mathrm{mmol})$ in ethanol $(50 \mathrm{~mL})$ containing catalytic amount of piperidine was heated under reflux for $4 \mathrm{~h}$. The reaction mixture was allowed to cool and poured into crushed ice then acidified with 
$\mathrm{HCl}$, The solid product so formed was collected by filtration and crystallized from the proper solvent.

2.8a 2-Amino-4-(4-chlorophenyl)-7-ethyl-5-methyl4H-pyrano[2,3-b]pyridine-3,6-dicarbonitrile (27a): Formed as pale yellow crystals (ethanol); $73 \%$. Yield; mp 100-102 ${ }^{\circ} \mathrm{C}$; IR (KBr) 3428, $3300\left(\mathrm{NH}_{2}\right), 2934$ (CH-aliph), $2206(\mathrm{CN}) \mathrm{cm}^{-1} ;{ }^{1} \mathrm{H}$ NMR $\left(\mathrm{CDCl}_{3}\right) \delta 1.26$ (s, 3H, Me), 1.72 (t, 3H, $J=5.5 \mathrm{~Hz}, \mathrm{Me}), 3.95$ (q, $\left.J=5.5 \mathrm{~Hz} 2 \mathrm{H}, \mathrm{CH}_{2}\right), 5.69$ (s, 1H, 4H-pyrane), 7.05$7.54\left(\mathrm{~m}, 6 \mathrm{H}\right.$, aromatic and $\left.\mathrm{NH}_{2}\right) ;{ }^{13} \mathrm{C}$ NMR $\left(\mathrm{CDCl}_{3}\right)$ $\delta 14.60,21.30,31.00,33.9077 .80,103.00,113.90$, $117.50119 .20,131.70,131.70,132.40,134.40,134.40$, 137.00, 148.45, 168.00, 173.20, 178.20. MS $(m / z) 350$ $\left(\mathrm{M}^{+}\right)$, Anal. Calcd. for $\mathrm{C}_{19} \mathrm{H}_{15} \mathrm{ClN}_{4} \mathrm{O}: \mathrm{C}, 65.05 ; \mathrm{H}$, 4.31; Cl. 10.11; N, 15.97; Found: C, 65.01; H, 4.28; Cl. $10.08 ; \mathrm{N}, 15.92$.

2.8b 2-Amino-4-(2-chlorophenyl)-7-ethyl-5-methyl4H-pyrano [2,3-b]pyridine-3,6-dicarbonitrile (27b): Formed as pale yellow crystals (ethanol); 89\% Yield; mp 106-108 ${ }^{\circ}$; IR (KBr) 3343, $3300\left(\mathrm{NH}_{2}\right), 2934$ (CH-aliph), $2196(2 \mathrm{CN}) \mathrm{cm}^{-1} ;{ }^{1} \mathrm{H}-\mathrm{NMR}\left(\mathrm{CDCl}_{3}\right) \delta$ $1.10(\mathrm{t}, 3 \mathrm{H}, J=5.5 \mathrm{~Hz}, \mathrm{Me}), 1.12(\mathrm{~s}, 3 \mathrm{H}, \mathrm{Me}), 3.47$ (q, $\left.J=5.5 \mathrm{~Hz} 2 \mathrm{H}, \mathrm{CH}_{2}\right), 5.00(\mathrm{~s}, 1 \mathrm{H}, 4 \mathrm{H}-$ pyrane), 7.11-8.27 (m, 6H, aromatic and $\left.\mathrm{NH}_{2}\right)$; $\mathrm{MS}(\mathrm{m} / z) 350$ $\left(\mathrm{M}^{+}\right)$, Anal. Calcd. for $\mathrm{C}_{19} \mathrm{H}_{15} \mathrm{ClN}_{4} \mathrm{O}: \mathrm{C}, 65.05 ; \mathrm{H}$, 4.31; Cl, 10.11; N, 15.97; Found: C, 65.12; H, 4.25; Cl. $10.00 ; \mathrm{N}, 15.85$.

\section{Results and discussions}

It has been found that 3 -aminopyridine 1 reacted readily with ethyl benzoylacetate 2 to give 3-oxo-3-phenyl-N- (pyridine-3-yl) propanamide 3. Establishing structure 3 was based on its elemental analysis and spectral data. Thus, ${ }^{1} \mathrm{H}-\mathrm{NMR}$ spectrum of compound $\mathbf{3}$ revealed the presence of a singlet signals at $\delta=4.14$ ppm corresponding to methylene group, a multiplet signal at $\delta=$ $7.24-8.70 \mathrm{ppm}$ corresponding to aromatic protons and singlet signal at $9.67 \mathrm{ppm}$ corresponding to $\mathrm{NH}$ group. The mass spectrum of $\mathbf{3}$ showed a molecular ion peak at $\mathrm{m} / \mathrm{z} 240\left(\mathrm{M}^{+}\right)$in agreement with its molecular formula $\mathrm{C}_{14} \mathrm{H}_{12} \mathrm{~N}_{2} \mathrm{O}_{2}$ and a number of fragments agree with the proposed structure. The IR spectrum of the same compound is in accordance with the proposed structure. Compound $\mathbf{3}$ underwent several chemical transformations via its reaction with a variety of electrophilic and nucleophilic reagents. Thus, when compound $\mathbf{3}$ is allowed to couple with aryl diazonium salts $\mathbf{4 a - d}$ affords the corresponding aryl hydrazones $\mathbf{5 a - d}$ based on their spectral data. For example, the ${ }^{1} \mathrm{H}$ NMR spectrum of $\mathbf{5 a}$ showed the presence of a multiple signal at $\delta=7.14-8.90 \mathrm{ppm}$ corresponding to aromatic protons and two signals at $\delta=11.77$ and 14.72 ppm corresponding to two $\mathrm{NH}$ groups, It should be emphasized here that the signal corresponding to amino function of hydrazo group appears at downfield at $\delta=14.72 \mathrm{ppm}$ due to the intramolecular hydrogen bonding with carbonyl group. ${ }^{30}$ The IR spectrum of the same product further supports the hydrozo structure. Fusion of aryl hydrazones 5a-d with malononitrile in domestic microwave oven for $3 \mathrm{~min}$ afforded the dihydropyridazines 7a-d via intermediacy of $\mathbf{6 a - d}$ (scheme 1). Formation of 7a is formed via losing $\mathrm{H}_{2} \mathrm{O}$ and subsequent cyclization of the intermediate 6a. Establishing structure $\mathbf{7}$ for the reaction of malononitrile with $\mathbf{5}$ was based on its elemental analysis and spectral data. For example, the ${ }^{1} \mathrm{H}$ NMR of $7 \mathbf{a}$ revealed the presence of a multiplet

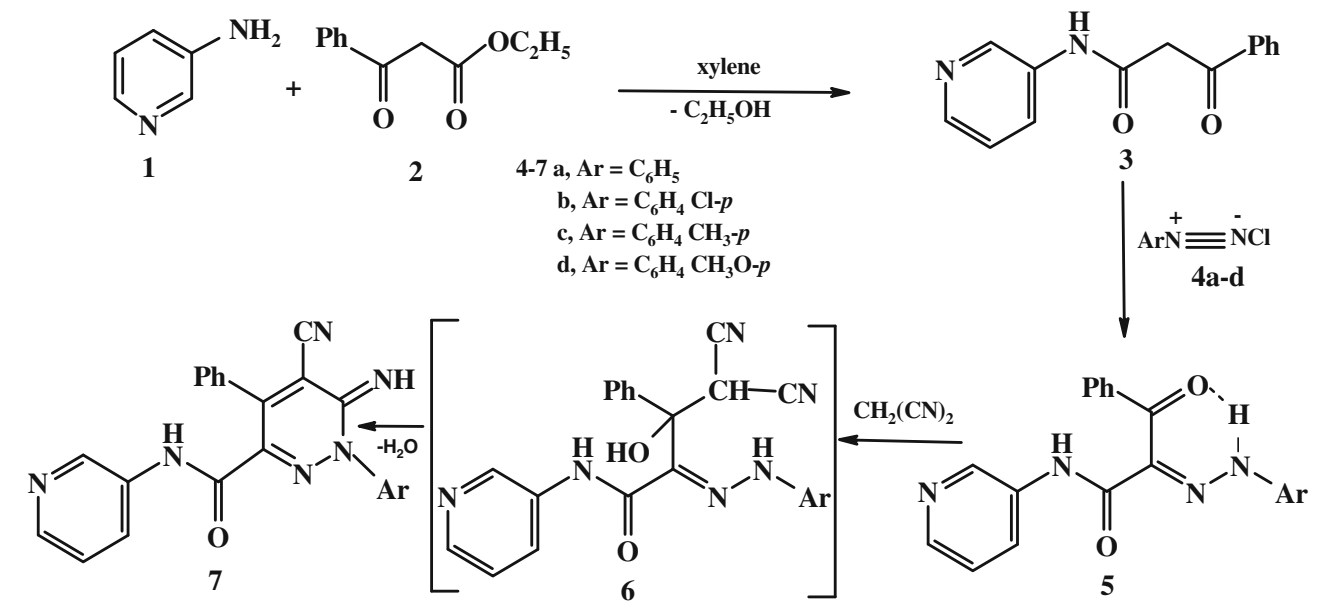

Scheme 1. Synthesis of dihydropyridazine derivatives 7a-d. 
signal at $\delta=7.26-8.01 \mathrm{ppm}$ corresponding to aromatic protons and $\mathrm{NH}$ group. The second $\mathrm{NH}$ group appeared as a singlet signal at $\delta=10.00 \mathrm{ppm}$. The mass spectrum of the same product is in accordance with the proposed structure. Thus, it showed a very intense molecular ion peak at $394\left(\mathrm{M}^{+}+2\right)$ and a number of fragments agree with the proposed structure.

Furthermore, the behaviour of $\mathbf{3}$ toward arylidenemalononitriles was also investigated. Thus, when 3oxo-3-phenyl-N-(pyridine-3-yl)propanamide 3 refluxed with arylidenemalononitrile $\mathbf{8 a}$ in ethanol containing catalytic amount of piperidine afforded the tetrahydropyridine 10a rather than its isomeric structure 12a based on its spectral data. For example, the 1H-NMR of compound 10a revealed the presence of two doublet signals at $\delta=4.25$ and $\delta=4.92 \mathrm{ppm}$ corresponding to methine protons at $\mathrm{C}-4$ and $\mathrm{C}-5$, a multiplet signal at $\delta=7.26-8.14 \mathrm{ppm}$ corresponding to aromatic protons and singlet signal at $\delta=8.78 \mathrm{ppm}$ corresponding to amino group. Compound 10a is believed to be formed via Michael type addition on the double bond system of $\mathbf{8 a}$ to give the non isolable interme- diate 9a that cyclizes in the same reaction condition to give 10a (scheme 2). Similarly, compound $\mathbf{3}$ reacted with arylidenemalononitriles $\mathbf{8 b} \mathbf{-} \mathbf{d}$ in refluxing ethanol to give tetrahydropyridine $\mathbf{1 0 b}-\mathbf{d}$ via intermediacy of 9b-d (scheme 2).

The foregoing results prompted us to investigate the behaviour of $\mathbf{3}$ towards some activated double bond such as 2-(ethoxymethylene)malononitrile 13a and ethyl 2-cyano-3-ethoxyacrylate 13b. Thus, when $\mathbf{3}$ is refluxed with 13a in ethanol containing a catalytic amount of piperidine, tetrahydropyridine 16a was obtained via intermediacy of the non isolable intermediates 14a and 15a (scheme 3). The structure of 16a was established on the basis of its elemental analysis and spectral data. For example, the ${ }^{1} \mathrm{H}-\mathrm{NMR}$ of $\mathbf{1 6 a}$ displayed a triplet and quartet signals at $\delta=1.55$ and $2.88 \mathrm{ppm}$ corresponding to methyl and methylene groups, respectively, two doublet signals at $\delta=$ $4.20 \mathrm{ppm}$ and $\delta=5.19 \mathrm{ppm}$ for the methine protons at C-4 and C-5 of the pyridine moiety, a multiplet signal of aromatic protons at $\delta=7.35-8.51 \mathrm{ppm}$ and a singlet signal at $\delta=8.61 \mathrm{ppm}$ characteristic

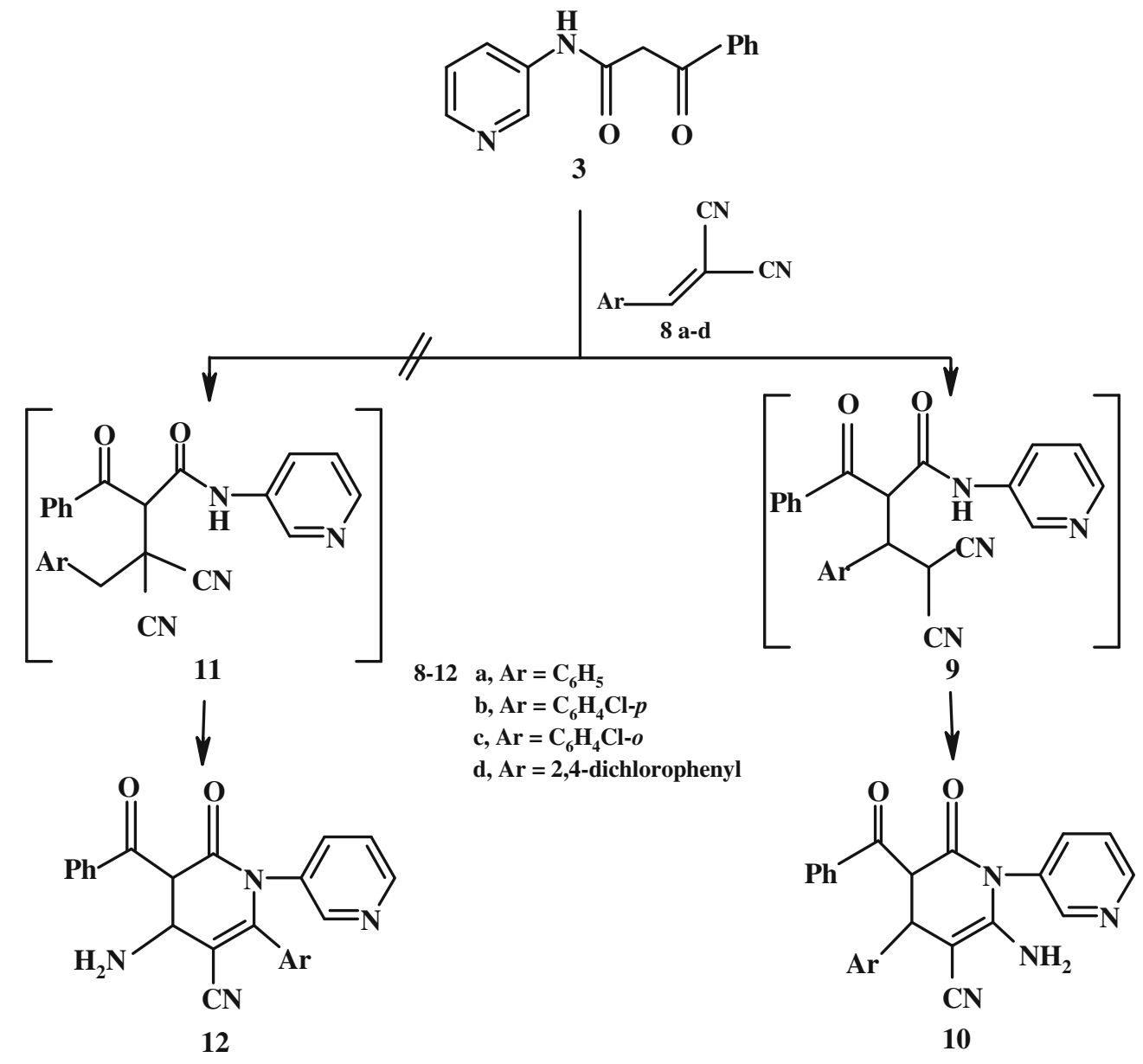

Scheme 2. Sythesis of tetrahydropyridine derivatives 10a-d. 
to amino group. The mass spectrum of compound $\mathbf{1 6 a}$ showed a molecular ion peak at m/z $362\left(\mathrm{M}^{+}\right)$corresponding to a molecular formula $\mathrm{C}_{20} \mathrm{H}_{18} \mathrm{~N}_{4} \mathrm{O}_{3}$. In the same manner, anilide 3 reacted with ethyl 2-cyano-3ethoxyacrylate $\mathbf{1 3 b}$ in refluxing ethanol piperidine solution to give tetrahydropyridine $\mathbf{1 6 b}$ via intermediacy of $14 \mathrm{~b}$ and $15 \mathrm{~b}$ (scheme 3). The identity of structure $\mathbf{1 6 b}$ was confirmed by its elemental analysis and spectral data.

The behaviour of enaminonitrile $\mathbf{1 7}$ with ethyl acetoacetate $\mathbf{1 8}$ in refluxing xylene afforded the dihydropyridine 21 via intermediacy of acyclic intermediates $\mathbf{1 9}$ and 20 that readily tautomerize and cyclized into $\mathbf{2 1}$ via losing water molecule (scheme 4). The structure of the latter product was established on the basis of its elemental analysis and spectral data. Thus, the ${ }^{1} \mathrm{H}$ NMR of $\mathbf{2 1}$ showed a singlet signal at $\delta=1.19 \mathrm{ppm}$ for the methyl group, a triplet signal at $\delta=1.29 \mathrm{ppm}$ corresponding to methyl group, a quartet signal at $\delta=2.66 \mathrm{ppm}$ characteristic to methylene group, and a broad signal at $\delta=4.99 \mathrm{ppm}$ due to $\mathrm{NH}$ function and a singlet signal at $\delta=6.10 \mathrm{ppm}$ corresponding to aromatic proton at C-3. The mass spectrum of the same product is in accordance with the proposed structure. Thus, it showed molecular ion peak at $164\left(\mathrm{M}^{+}+2\right)$ and a number of fragments agrees with the proposed structure.

The synthetic potentiality of compound $\mathbf{2 1}$ towards a variety of electrophilic reagents and aryl diazonium salts was also investigated. Thus, when $\mathbf{2 1}$ is allowed to couple with aryl diazonium salts $\mathbf{4 b}$ a compound with molecular formula $\mathrm{C}_{15} \mathrm{H}_{13} \mathrm{ClN}_{4} \mathrm{O}=300$ was obtained. This was considered to be the hydrazo compound 22 or its tautomeric structure 23. Structure 23 was ruled out and structure 22 was established for this reaction product based on its elemental analysis and spectral data. For example, the proton ${ }^{1} \mathrm{H}$ NMR revealed the presence of a signal corresponding to amino function at higher field $\delta=8.01 \mathrm{ppm}$. On the other hand, if the reaction product is $\mathbf{2 3}$ one would expect that amino function to appear at much lower field (scheme 4). Similarly, compound 21 coupled with aryl diazonium salt $\mathbf{4 c}$ to give $\mathbf{2 2 b}$ based on its spectral analyses. The behaviour of $\mathbf{2 1}$ toward arylidenemalononitrile reagents was also investigated. Thus, treatment of $\mathbf{2 1}$ with arylidenemalononitrile $\mathbf{8 b}$ in ethanol piperidine solution afforded a product identified as 2-amino-4-(4-chlorophenyl)-7-ethyl-5-methyl4H-pyrano[2,3-b]pyridine-3,6-dicarbonitrile (27a).

Formation of 27a from 21 and arylidenemalononitrile $\mathbf{8 b}$ is believed to be formed via Michael type addition of compound $\mathbf{2 1}$ on the double bond system of $\mathbf{8 b}$ to give the acyclic intermediate $\mathbf{2 4 a}$ which is then underwent cyclization and subsequent tautomerism to give 27a as demonstrated in scheme 4 . The structure of the latter product was established on the basis of its elemental analysis and spectral data. Similarly, 21 reacted with arylidenemalononitrile $\mathbf{8 c}$ in the same reaction condition to afford pyranopyridine $\mathbf{2 6 b}$ (scheme 4 ). The

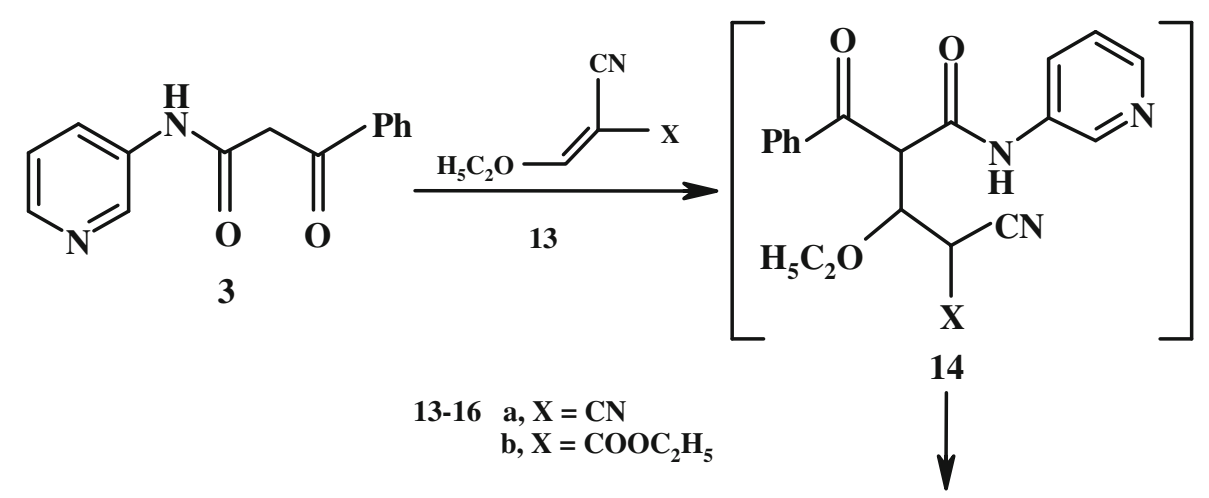<smiles>[Y]C1=C(N)N(c2cccnc2)C(=O)C(C(=O)c2ccccc2)C1OC</smiles><smiles>[X]C1C(=N)N(c2cccnc2)C(=O)C(C(=O)c2ccccc2)C1OCC</smiles>

Scheme 3. Synthesis of tetrahydropyridine derivative $\mathbf{1 6 a}, \mathbf{b}$. 


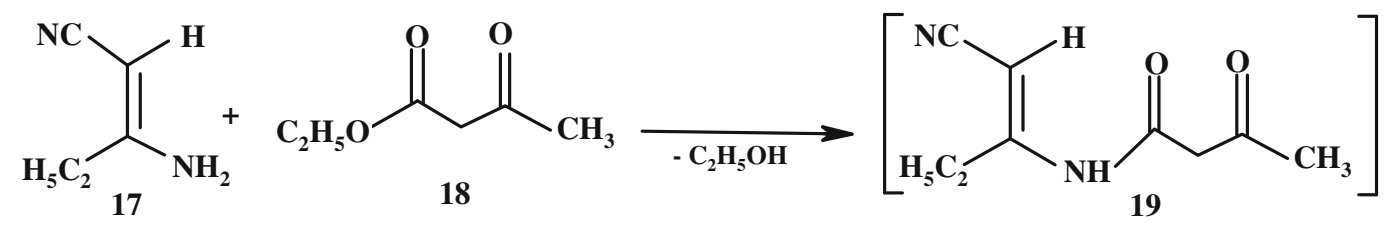

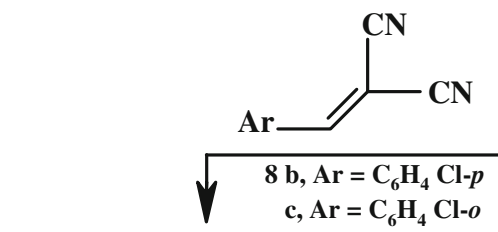<smiles>Cc1[nH]c(=O)c(C([Al])C(C#N)C#N)c(C)c1C#N</smiles><smiles>[V][V]</smiles>

25<smiles>[3H]I</smiles>

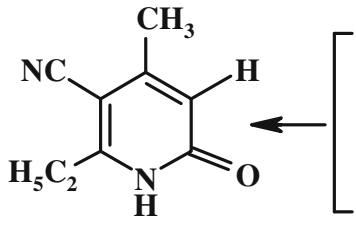

21

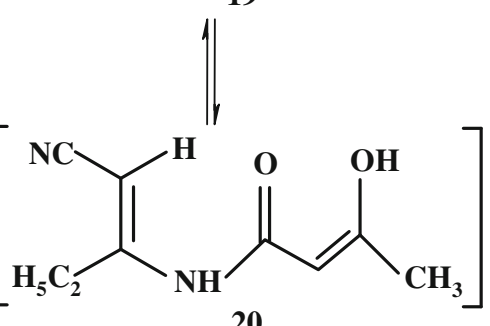

20

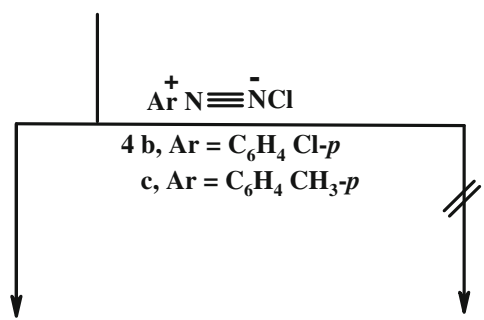

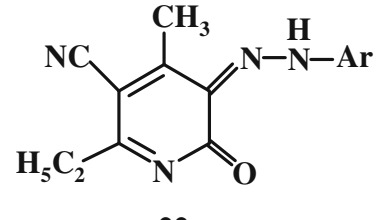

22

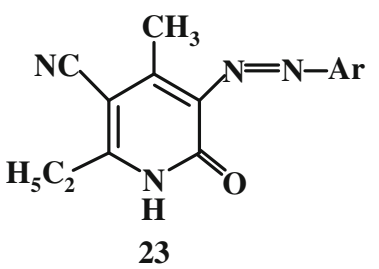

$$
\begin{aligned}
22,23 \mathrm{a}, \mathrm{Ar} & =\mathrm{C}_{6} \mathrm{H}_{4} \mathrm{Cl}-p \\
\mathrm{~b}, \mathrm{Ar} & =\mathrm{C}_{6} \mathrm{H}_{4} \mathrm{CH}_{3}-p \\
24-27 \mathrm{a}, \mathrm{Ar} & =\mathrm{C}_{6} \mathrm{H}_{4} \mathrm{Cl}-p \\
\mathrm{~b}, \mathrm{Ar} & =\mathrm{C}_{6} \mathrm{H}_{4} \mathrm{Cl}-\rho
\end{aligned}
$$

Scheme 4. Synthesis of pyranopyridine derivatives $27 \mathbf{a}, \mathbf{b}$.

identity of the product $\mathbf{2 7} \mathbf{b}$ was established on the basis of elemental analyses and spectral data.

\section{Conclusion}

The synthesis of a number of new hydrazo, dihydropyridazine, tetrahydropyridine, dihydropyridine and pyranopyridine derivatives with expected biological activities was achieved by utilizing the chemistry of propanamide $\mathbf{3}$ and enaminonitrile $\mathbf{1 7}$.

\section{Acknowledgement}

The authors are grateful to Prof. A E Khodair, Department of Chemistry, Faculty of Science, Suez Canal
University, Ismailia, Egypt, for valuable support and improving this manuscript.

\section{References}

1. (a) Teague S J 2008 J. Org. Chem. 73 9765; (b) Movassaghi M, Hill M D and Ahmad O K J 2007 Am. Chem. Soc. 12910096

2. Amr A E, Abo-Ghalia M and Abdalah M M 2006 Z. Naturforsch. 61b 1335

3. Amr A E, Mohamed A M and Ibrahim A A 2003 Z. Naturforsch. 58b 861

4. Amr A E, Mohamed A M, Mohamed S F, Abdel-Hafez N A and Hammam A G 2006 Bioorg. Med. Chem. 14 5481

5. Zhou Y, Kijima T, Kuwahara S, Watanabe M and Izumi T 2008 Tetrahedron Lett. 493757 
6. Franco L H, Joffe E B K, Puricelly L, Tatian M, Seldes A M and Palermo J A 1998 J. Nat. Prod. 611130

7. (a) Farhanullah A N, Goel A and Ram V J 2003 J. Org. Chem. 68 2983; (b) Joule J A, Smith G and Mills K 1995 Heterocyclic chemistry (London: Chapman and Hall) 3rd edn., pp. 72

8. Oka Y, Omura K, Miyake A, Itoh K, Tomimoto M, Tada N and Yurugi S 1975 Chem. Pharm. Bull. 232239

9. Matsuura I, Yoneda F and Nitta Y 1966 Chem. Pharm. Bull. 141010

10. Kricka L 1996 Pure Appl. Chem. 68 (I0), 1825

11. Sivakumar R, Anbalagan N, Vedachalam G and Joseph T L 2003 Biol. Pharm. Bull. 26(10) 1407

12. Nagwade R R, Khanna V V, Bhagwat S S and Shinde D B 2005 Eur. J. Med. Chem. 401325

13. Sacchi A, Laneri S, Arena F, Abigente E, Gallitelli M, D'amico M, Filippelli W and Rossi F 1999 Eur. J. Med. Chem. 341003

14. Park E H and Park M S vatives 2007 J. Kor. Chem. Soc. 51(3) 244

15. Frank H and Heinisch G 1990 Pharmacologically active Pyridazines Part I; In Progress in medicinal chemistry, G P Ellis, G B West (eds) Amsterdam: Elsevier 27, 1

16. Frank H and Heinisch G 1992 Pharmacologically active Pyridazines Part II; In Progress in medicinal chemistry, G P Ellis, D K Luscombe (eds) Amsterdam: Elsevier 29, 141

17. Rollas S, Gülerman N and Erdeniz H 2002 Farmaco 57 171
18. Küçükgüzel Ş G, Rollas S, Küçükgüzel İ and Kiraz M 1999 Eur. J. Med. Chem. 341093

19. Küçükgüzel Ş G, Oruç E E, Rollas S, Şahin F and Özbek A 2002 Eur. J. Med. Chem. 37197

20. Kaymakçıŏlu K B, Oruç E E, Unsalan S, Kandemirli F, Shvets N, Rollas S and Anatholy D 2006 Eur. J. Med. Chem. 411253

21. Todeschini A R, Miranda A L, Silva C M, Parrini S C and Barreiro E 1998 J. Eur. J. Med. Chem. 33189

22. Fraga A G M, Rodrigues C R, Miranda A L P, Barreiro E J and Fraga C A M 2000 Eur. J. Pharm. Sci. 11 285

23. Walcourt A, Loyevsky M, Lovejoy D B, Gordeuk V R and Richardson D R 2004 Int. J. Biochem. Cell Biol. 36 401

24. Gemma S, Kukreja G, Fattorusso C, Persico M, Romano M, Altarelli M, Savini L, Campiani G, Fattorusso E and Basilico N 2006 Bioorg. Med. Chem. Lett. 165384

25. Küçükgüzel Ş G, Rollas S, Erdeniz H and Kiraz M 1999 Eur. J. Med. Chem. 34153

26. Abdel Hafiz I S 2000 Z. Naturforsch. 55b 321

27. Abdel Hafiz I S, Darwish E S and Mahmoud F F 1999 J. Chem. Res. (M). 3254

28. Gaber H M, Abdel Hafiz I S, ElSawy K M and Sherif M S 2010 Acta Chim. Slov. 57

29. Hussein A M, Ishak E A, Abdel Hafiz I S and Elnagdi M H 2007 Phosphorus, Sulfur, Silicon. 1822897

30. Hafiz A, Ibrahim S, Ramiz M M M and Sarhan A A M 2011 Chin. J. Chem. 291154 